International Journal of Modern Physics A

(C) World Scientific Publishing Company

\title{
OBSERVATION OF REDUCTION IN CASIMIR FORCE WITHOUT CHANGE OF DIELECTRIC PERMITTIVITY
}

\author{
A. A. BANISHEV, C.-C. CHANG, R. CASTILlO-GARZA, \\ Department of Physics and Astronomy, University of California, Riverside, CA 92521, USA \\ G. L. KLIMCHITSKAYA \\ North-West Technical University, Millionnaya Street 5, St.Petersburg, 191065, Russia \\ V. M. MOSTEPANENKO \\ Noncommercial Partnership "Scientific Instruments", Tverskaya Street 11, Moscow, 103905, \\ Russia \\ U. MOHIDEEN \\ Department of Physics and Astronomy, University of California, Riverside, CA 92521, USA \\ Umar.Mohideen@ucr.edu \\ Received 17 November 2011 \\ Revised 4 January 2012
}

\begin{abstract}
Additional information is provided on the effect of the significant (up to $35 \%$ ) reduction in the magnitude of the Casimir force between an Au-coated sphere and an indium tin oxide film which was observed after UV treatment of the latter. A striking feature of this effect is that the reduction is not accompanied with a corresponding variation of the dielectric permittivity, as confirmed by direct ellipsometry measurements. The measurement data are compared with computations using the Lifshitz theory. It is shown that the data for the untreated sample are in a very good agreement with theory taking into account the free charge carriers in the indium tin oxide. The data for the UV-treated sample exclude the theoretical results obtained with account of free charge carriers. These data are found to be in a very good agreement with theory disregarding the free charge carriers in an indium tin oxide film. A possible theoretical explanation of our observations as a result of phase transition of indium tin oxide from metallic to dielectric state is discussed in comparison with other related experiments.
\end{abstract}

Keywords: Casimir force; dielectric permittivity; charge carriers.

PACS numbers: 78.20.-e, 78.66.-w, 12.20.Fv, 12.20.Ds

\section{Introduction}

In the last few years experiments on the Casimir effect have been numerous and varied. They deal with different materials, such as metals, semiconductors and dielectrics, and with the influence of phase transitions on the Casimir force ${ }^{1-4}$ Many experiments tried to significantly reduce the magnitude of the Casimir force, as 
compared $-\sqrt{7}$ to two test bodies made of good metal, such as Au. This was achieved by measuring the Casimir force between an $\mathrm{Au}$ sphere and a plate made of different semiconductors, $\frac{8}{-12}$ semimetals, $\frac{13}{2}$ and when the plate materials undergo transformation from metallic to dielectric state ${ }^{14 \mid 15}$ or from crystalline to amorphous phase ${ }^{[16}$ In all the cases considered so far any detectable changes in the magnitude of the Casimir force were accompanied by significant variations in the dielectric permittivity of the plate over a wide region of imaginary frequencies. This observation is commonly considered as a natural and invariable consequence of the Lifshitz theory! 117

Here, we report additional information on a significant (up to 35\%) reduction in the magnitude of the Casimir force between an Au-coated sphere and an indium tin oxide (ITO) film recently observed $\frac{18}{18}$ wich occurs after UV treatment of the ITO. A striking feature of this phenomenon is that the reduction in the force magnitude is not accompanied with corresponding variations in the dielectric permittivity of ITO under UV treatment. This is confirmed by the direct ellipsometry measurements of the imaginary parts of the dielectric permittivity for both the untreated and UVtreated samples.

ITO $\left(\mathrm{In}_{2} \mathrm{O}_{3}: \mathrm{Sn}\right)$ has long been suggested $\frac{19}{19}$ for use in Casimir physics due to its unique electric properties. It was measured 1112 that the gradient of the Casimir force between an Au sphere and an ITO plate is roughly 40\%-50\% smaller than between an $\mathrm{Au}$ sphere and an Au plate. The same rate of a decrease, as compared to the case of $\mathrm{Au}-\mathrm{Au}$ test bodies, is confirmed by us 18 for the magnitude of the Casimir force between an Au sphere and an untreated ITO sample. The overall decrease for a UV-treated sample is up to $65 \%$ in comparison to Au-Au surfaces.

All figures presented in this paper use the experimental data of the second set of our measurements performed before and after the UV treatment (in previously published paper ${ }^{18}$ only the first measurement set has been used). Here we report for the first time the experimental data for the total measured force (electric plus Casimir) and perform detailed comparison between experiment and theory over a narrower separation region from 60 to $150 \mathrm{~nm}$ where the measurement data are the most precise. The measurement data for the untreated sample are found to be in a very good agreement with the Lifshitz theory when the contribution of free charge carriers in the ITO is taken into account. For the UV-treated sample, the measurement data agree very well with computations disregarding free charge carriers in the ITO film. These data are in complete disagreement with theory taking into account the contribution of free charge carriers in the dielectric permittivity of ITO. We discuss in detail a possible theoretical explanation for the observed new effect of reduction in the Casimir force without change of dielectric permittivity. We explain this effect by the phase transition from metallic to dielectric state which occurs in the ITO film under the influence of UV treatment. We compare our measurements with the results of other experiments (already performed and proposed) where the magnitude of the Casimir force was changed as a result of the phase transition. 
The paper is organized as follows. In Sec. 2 we present the experimental results for the Casimir force, the dielectric permittivity of ITO and the surface roughness. Section 3 contains comparison between experiment and theory. Theoretical explanation of the effect of reduction in Casimir force without change in dielectric permittivity is considered in Sec. 4 . Section 5 contains our conclusions and discussion.

\section{Experimental Results}

We used a modified multimode atomic force microscope (AFM) to measure the total force (electrostatic plus Casimir) between an Au-coated polystyrene sphere and an ITO film deposited on a quartz plate. Measurements were performed at a pressure of $10^{-6}$ Torr at $2^{\circ} \mathrm{C}$. The radius of the Au-coated sphere was measured to be $R=101.2 \pm 0.5 \mu \mathrm{m}$. The Au coating was done in an oil free thermal evaporator at a very low deposition rate of $3.37 \AA / \mathrm{min}$. The thickness of the Au layer was $105 \pm 1 \mathrm{~nm}$. The ITO film was prepared by RF sputtering (Thinfilm Inc.). The thickness and nominal resistivity of the ITO film were measured to be $74.6 \pm 0.2 \mathrm{~nm}$ and $42 \Omega$ sq, respectively. The ITO sample was cleaned in an ultrasonic bath with acetone then with methanol and finally with ethanol alternated with rinsing in DI water, and finally dried in a flow of nitrogen gas.

After the force measurements for the untreated ITO sample were completed, it was UV-treated for 12 hours in a special chamber containing a UV lamp. The spectrum of this lamp has the primary peak at $254 \mathrm{~nm}\left(5.4 \mathrm{~mW} / \mathrm{cm}^{2}\right.$ at $1.9 \mathrm{~cm}$ distance) and a secondary peak at $365 \mathrm{~nm}\left(0.2 \mathrm{~mW} / \mathrm{cm}^{2}\right.$ at the same distance). After the UV treatment the sample was cleaned as described above. To stabilize the laser used for the detection of deflection of the AFM cantilever, we employed a liquid nitrogen cooling system, which maintained the temperature at $2^{\circ} \mathrm{C}$.

\subsection{Measurement of the Casimir force between an Au sphere and an ITO plate}

The scheme of force measurements using an AFM is as follows! $12|4| 5 \mid 8-16$ An Au-coated cantilever of an AFM with attached sphere undergoes a deflection $z$ in response to the sphere-plate force $F_{\text {tot }}$ in accordance with Hooke's law $F_{\text {tot }}=k z$, where $k$ is the spring constant. The photodetectors measure this deflection through differential measurement of the laser beam intensity reflected from the cantilever. The respective deflection signal $S_{\text {def }}$ as a function of separation distance $a$ between the sphere and the plate leads to a force-distance curve. This signal can be calculated according to $z=m S_{\text {def }}$ and measured in volts, where $m$ measured in $\mathrm{nm} / \mathrm{V}$ is the cantilever deflection per unit photodetector signal, also called the deflection coefficient.

Due to the presence of surface roughness, there is some nonzero minimum separation $z_{0}$ that can be achieved when the sphere and the plate are approaching. It is called the separation on contact. Taking into account deflection of the cantilever 
under the influence of the total force, the absolute separation between the sphere and the plate is given by

$$
a=z_{0}+z_{\text {piezo }}+m S_{\text {def }}
$$

Here, $z_{\text {piezo }}$ is the distance traveled by the plate attached to the top of the piezoelectric actuator which was calibrated interferometrically $20 \mid 21$ To change the separation, a continuous triangular voltage was applied to the actuator. As a result, an ITO plate was moved towards the Au sphere starting at maximum separation of $2 \mu \mathrm{m}$ until the contact, and the cantilever deflection was recorded at every $0.2 \mathrm{~nm}$.

The experimental Casimir force was found from the equation

$$
F(a)=F_{\text {tot }}\left(a, V_{i}\right)-F_{\text {el }}\left(a, V_{i}\right)=\tilde{k} S_{\text {def }}\left(a, V_{i}\right)-F_{\text {el }}\left(a, V_{i}\right),
$$

where $V_{i}(i=1,2, \ldots, 10)$ are voltages applied to the ITO plate while the sphere remained grounded, and $\tilde{k}=k m$. The voltage supply used had a $1 \mu \mathrm{V}$ resolution, and special measures were undertaken to prevent surge currents and to reduce electrical noise. The electric force between a conducting plate and a conducting sphere takes the form 122

$$
F_{\mathrm{el}}\left(a, V_{i}\right)=X(a)\left(V_{i}-V_{0}\right)^{2},
$$

where $V_{0}$ is the residual potential difference due to different work functions of the sphere and plate materials. $X(a)$ is the known function of $a$ and $R$. In the wide range of separations it can be presented with less than $0.01 \%$ error in the polynomial form 9

$$
X(a)=-2 \pi \epsilon_{0} \sum_{i=-1}^{6} c_{i}\left(\frac{a}{R}\right)^{i},
$$

where $c_{i}$ are numerical coefficients $\underline{9}$

Equations (10)-(4) can be used to perform the electrostatic calibrations, i.e., to determine the values of $V_{0}, m, z_{0}$ and $\tilde{k}$ by applying voltages $V_{i}$ to the ITO film and by measuring the respective deflection signal $S_{\text {def }}$. The calibration process is discussed in detail in Refs. 18, 23 and 24. From the tops of the parabolic dependences of $S_{\text {def }}$ on $V_{i}$ the mean values of $V_{0}=-196.8 \pm 1.5 \mathrm{mV}$ and $V_{0}=64.8 \pm 2.0 \mathrm{mV}$ for the untreated and UV-treated samples, respectively, were determined in the second measurement set considered here. To find the mean values of $V_{0}$, we first determined the individual $V_{0}$ and found that they are separation-independent in the limits of random errors if the corrections due to mechanical drift of the sphere-plate separation and to finiteness of the data acquisition rate are introduced. Thus, our measurements are free of anomalies discussed in the literature $25-28$ and do not support the existence in our setup of large electric fields due to patch effect, 29 surface contaminants etc. in addition to the electrostatic force (3). Next, from the curvatures of parabolas the mean values of other parameters were determined related to the second measurement set: $m=104.4 \pm 0.5 \mathrm{~nm} / \mathrm{V}, z_{0}=29.6 \pm 0.5 \mathrm{~nm}, \tilde{k}=1.51 \pm 0.02 \mathrm{nN} / \mathrm{V}$ for the untreated sample and $m=104.2 \pm 0.6 \mathrm{~nm} / \mathrm{V}, z_{0}=29.0 \pm 0.6 \mathrm{~nm}, \tilde{k}=1.51 \pm 0.02 \mathrm{nN} / \mathrm{V}$ 

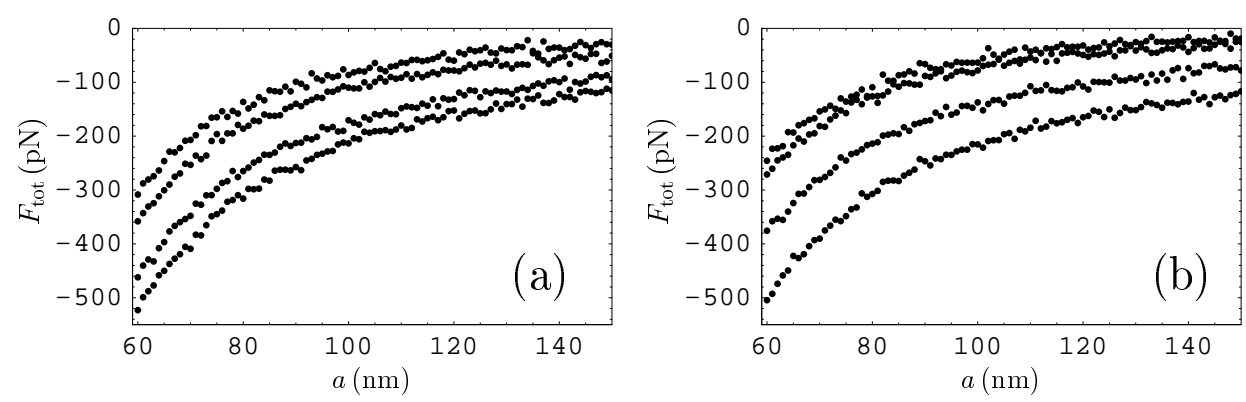

Fig. 1. Total measured forces as functions of separation with different applied voltages are indicated as dots for (a) the untreated and (b) UV-treated sample (see text for further discussion).

for the UV-treated sample. The error bars in the values of all the above parameters are indicated at a $95 \%$ confidence level.

The total force $F_{\text {tot }}$ as a function of separation was measured 10 times with a step of $0.2 \mathrm{~nm}$ for each of the 10 different voltages applied to the plate. Note that only the interpolated data at $1 \mathrm{~nm}$ step were analyzed. As an example in Fig. 1 we demonstrate four typical force-distance curves from bottom to top (a) for the untreated sample with applied voltages $-265 \mathrm{mV},-255 \mathrm{mV},-230 \mathrm{mV},-200 \mathrm{mV}$, respectively, and (b) for the UV-treated sample with respective applied voltages equal to $140 \mathrm{mV}, 120 \mathrm{mV}, 90 \mathrm{mV}$, and $70 \mathrm{mV}$. As can be seen in Fig. 1(a,b), for the largest magnitude of applied voltages (bottom curves) the total measured forces are rather close in magnitude. For the applied voltages $-265 \mathrm{mV}$ and $140 \mathrm{mV}$ the magnitudes of $V-V_{0}$ are equal to approximately 68 and $75 \mathrm{mV}$ for the untreated and UV-treated samples, respectively. These give major contributions to the magnitudes of the total force. Thus, without detailed analysis (see Sec. 3) it is not possible to make any conclusion about the magnitudes of the Casimir force. A different situation arises with the top curves in Fig. 1(a,b) [the applied voltages are equal to (a) $-200 \mathrm{mV}$ and (b) $70 \mathrm{mV}]$. Here $\left|V-V_{0}\right|$ is equal to only 3 and $5 \mathrm{mV}$ for the untreated and UV-treated samples, respectively. The magnitudes of the respective electric forces are rather small in comparison with still large (and quite different) magnitudes of the total forces shown by the top curves in Fig. 1(a,b). This suggests that the Casimir forces from the untreated and UV-treated samples giving major contributions to the total forces shown by the top curves are significantly different (a conclusion confirmed in Sec. 3).

Using Eqs. (2)-(4), at each separation 100 values of the Casimir force were obtained shown in Fig. 2(a) by the dark-grey and light-grey dots with a step of $2 \mathrm{~nm}$ for the untreated and UV-treated sample, respectively. The respective black solid lines show the mean Casimir forces averaged over 100 repetitions. From Fig. 2(a) it is seen that the UV treatment results in a significant decrease in the magnitude of the Casimir force from $21 \%$ to $35 \%$ depending on separation. In Fig. 2(b) we present a histogram for the measured Casimir force at $a=80 \mathrm{~nm}$ for the untreated sample (right) and UV-treated sample (left). The corresponding Gaussian distributions are 

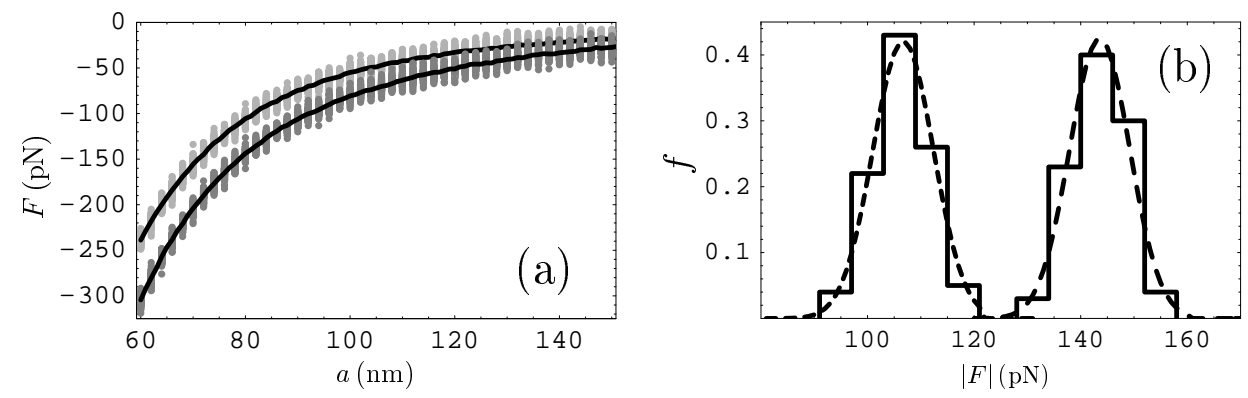

Fig. 2. (a) Mean measured Casimir forces as a function of separation are shown by the lower and upper solid lines for the untreated and UV-treated sample, respectively. Respective 100 individual values of the force are shown as dark-grey and light-grey dots at $2 \mathrm{~nm}$ intervals. (b) The histograms for measured Casimir force for the untreated (right) and UV-treated (left) sample at $a=80 \mathrm{~nm} . f$ is the fraction of 100 data points having the force values in the bin indicated by the vertical lines.

shown by the dashed lines. They are characterized by the mean Casimir forces equal to $F=-143.7 \mathrm{pN}$ and $F=-105.5 \mathrm{pN}$ for the untreated and UV-treated From Fig. 2(b) it is seen that the Gaussian distributions for the untreated and UVtreated samples do not overlap lending great confidence to the effect of reduction of the Casimir force. From the lower solid line in Fig. 2(a) one can conclude also that the use of an untreated ITO plate leads to a $40 \%-50 \%$ decrease in the force magnitude in comparison with the case of two Au bodies in agreement with previous work 1112 where a similar result was obtained for the Casimir pressure.

In Fig. 3(a,b) the mean measured Casimir forces are shown as crosses for the untreated and UV-treated samples, respectively. The arms of the crosses indicate the total experimental errors in $a$ and $F$ calculated at a $95 \%$ confidence level. The error in $a$ is mostly determined by the error in $z_{0}$ indicated above. The total experimental errors in the Casimir force, $\Delta F$, as a function of $a$, are shown by the solid lines in the insets to Fig. 3(a,b). They are obtained by adding in quadrature the random and systematic erros shown in the insets by the short-dashed and long-
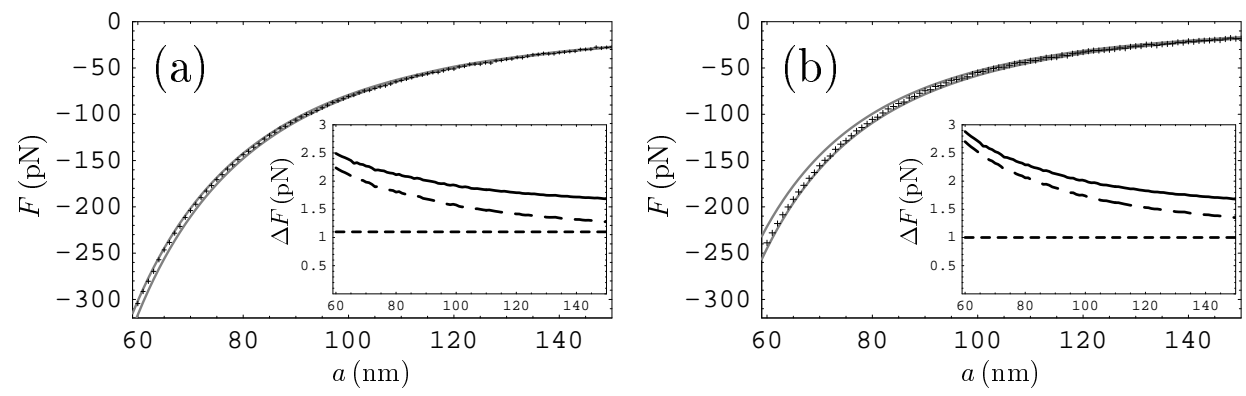

Fig. 3. The mean measured Casimir force indicated as crosses and the theoretical Casimir force shown by the pairs of solid lines as functions of separation for (a) the untreated (free charge carriers are included) and (b) UV-treated sample (free charge carriers are omitted). In the insets the short-dashed, long-dashed and solid lines show the random, systematic and total experimental errors, respectively, for the same samples as in the main fields. 
dashed lines, respectively. As can be seen in the insets, the random errors do not depend on separation. The systematic errors shown in the insets to Fig. 3(a,b) were found as a combination of the systematic error in the total measured force and the error in the electrostatic force subtracted in accordance with Eq. (2). In its turn, the systematic error in the total measured force is determined by the instrumental noise including the background noise level, and the errors in calibration. The error in the calculation of the electric force plays the role of a systematic error with respect to the Casimir force obtained from Eq. (2). It is mostly determined by the error in the measurement of separations. In fact the error in the electric force depends on the applied voltage (to obtain the long-dashed lines in the insets, the mean values of this error averaged over 10 applied voltages were used).

\subsection{Investigation of the dielectric permittivity of ITO and surface roughness}

The optical properties of ITO depend on a layer composition, thickness and other parameters. Previous experiments $11 / 12$ used the parametrization of the dielectric permittivity of ITO obtained ${ }^{30}$ with the help of the Tauc-Lorentz mode 31 and the Drude model. This did not lead to good agreement between experiment and theory. For this experiment the imaginary parts of the dielectric permittivity of ITO were determined by means of ellipsometry ${ }^{32}$ for both untreated and UV-treated samples. The region of frequencis from 0.04 to $8.27 \mathrm{eV}$ was covered by the IR-VASE and VUVVASE ellipsometers. The obtained results are shown in Fig. 4(a,b) by the solid lines. (Note that the ITO resistivity decreases with depth leading to the so-called top and bottom dielectric permittivities differing in the region $\omega<0.5 \mathrm{eV}$.) In the region of frequencies $\omega<0.04 \mathrm{eV}$ the measured top $\operatorname{Im} \varepsilon^{(\text {ITO })}$ which was found to lead to a good agreement with measured Casimir forces was extrapolated using the imaginary part of the Drude model with the plasma frequency $\omega_{p}=1.5 \mathrm{eV}$ and the relaxation parameter $\gamma=0.128 \mathrm{eV}$ (for the untreated sample) and $\omega_{p}=1.5 \mathrm{eV}, \gamma=0.132 \mathrm{eV}$ (for the UV-treated sample). For comparison purposes the dielectric permittivity 30
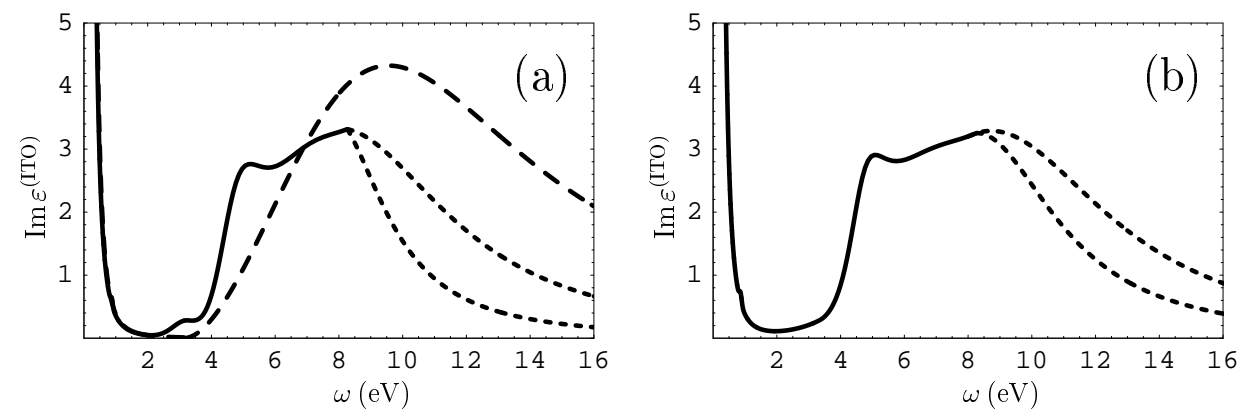

Fig. 4. The imaginary parts of dielectric permittivity of ITO measured by ellipsometry as functions of frequency are shown by the solid lines for (a) the untreated and (b) UV-treated sample. The short-dashed lines present possible extrapolations of the measured data to higher frequencies. The long-dashed line presents the results of Ref. 30. 
8 A. A.Banishev et al.

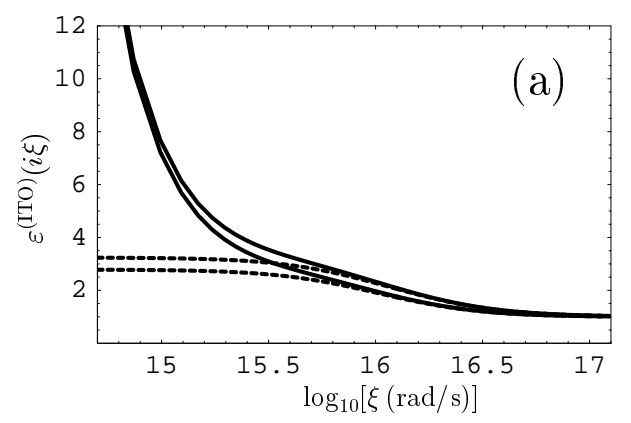

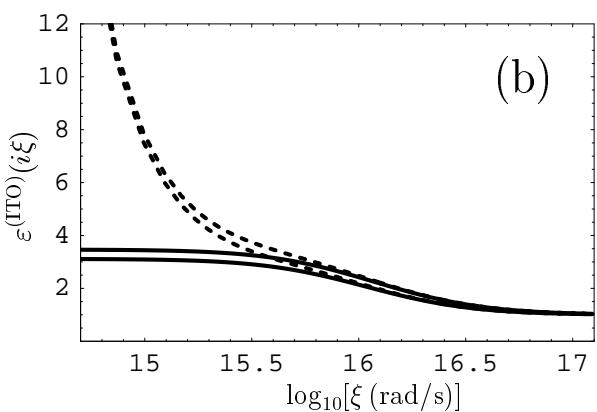

(b)

Fig. 5. The dielectric permittivity of ITO as functions of imaginary frequency for (a) the untreated and (b) UV-treated sample. The two solid and two dashed lines are obtained with different extrapolations of the ellipsometry data to higher frequencies shown by the short-dashed lines in Fig. 4(a,b). The solid and dashed lines correspond to (a) included and omitted contribution of free charge carriers, respectively, and (b) omitted and included contribution of free charge carriers, respectively.

is shown in Fig. 4(a) by the long-dashed line.

The extrapolation of the measured data to higher frequencies (up to $16 \mathrm{eV}$ ) is also required keeping in mind that the Casimir force was measured at rather short separations $a \geq 60 \mathrm{~nm}$. This was done by means of the oscillator function

$$
\operatorname{Im} \varepsilon^{(\mathrm{ITO})}(\omega)=\frac{g_{0} \gamma_{0} \omega}{\left(\omega^{2}-\omega_{0}^{2}\right)^{2}+\gamma_{0}^{2} \omega^{2}} .
$$

For the untreated sample, the reasonable smooth extrapolations are bounded between the short-dashed lines in Fig. 4(a) which are described by Eq. (5) with the parameters $g_{0}=240.54 \mathrm{eV}^{2}, \gamma_{0}=8.5 \mathrm{eV}, \omega_{0}=9.0 \mathrm{eV}$ (the upper line) and $g_{0}=111.52 \mathrm{eV}^{2}, \gamma_{0}=4.0 \mathrm{eV}, \omega_{0}=8.0 \mathrm{eV}$ (the lower line). For the UV-treated sample [Fig. 4(b)] the corresponding parameters are $g_{0}=280.28 \mathrm{eV}^{2}, \gamma_{0}=9.2 \mathrm{eV}$, $\omega_{0}=9.8 \mathrm{eV}$ (the upper short-dashed line) and $g_{0}=128.28 \mathrm{eV}^{2}, \gamma_{0}=4.5 \mathrm{eV}$, $\omega_{0}=8.8 \mathrm{eV}$ (the lower short-dashed line). From Fig. 4(a,b) it is seen that the UV treatment does not lead to any significant changes in the $\operatorname{Im} \varepsilon^{(\mathrm{ITO})}(\omega)$. Using the Kramers-Kronig relation, we have obtained $\varepsilon^{(\mathrm{ITO})}(i \xi)$ for both the untreated and UV-treated samples. The results are shown by the pairs of solid and dashed lines in Figs. 5(a) and 5(b) for the untreated and UV-treated samples, respectively. Each pair of lines corresponds to the pair of short-dashed lines in Fig. 4(a,b). The dashed lines in Fig. 5(a) [solid lines in Fig. 5(b)] present $\varepsilon^{(\mathrm{ITO})}(i \xi)$ with the contribution of free charge carriers omitted. From Fig. $5(\mathrm{a}, \mathrm{b})$ it is also seen that $\varepsilon^{(\mathrm{ITO})}(i \xi)$ is scarcely affected by the UV treatment.

The averaged dielectric permittivity of the quartz plate underlying the ITO film was described in the Ninham-Parsegian approximation

$$
\varepsilon^{(Q)}(i \xi)=1+\frac{C_{\mathrm{IR}}}{1+\frac{\xi^{2}}{\omega_{\mathrm{IR}}^{2}}}+\frac{C_{\mathrm{UV}}}{1+\frac{\xi^{2}}{\omega_{\mathrm{UV}}^{2}}}
$$

with the parameters ${ }^{33} C_{\mathrm{IR}}=1.93, C_{\mathrm{UV}}=1.359, \omega_{\mathrm{IR}}=0.1378 \mathrm{eV}$, and $\omega_{\mathrm{UV}}=$ $13.38 \mathrm{eV}$. As to $\varepsilon^{(\mathrm{Au})}(i \xi)$, it was obtained by means of the Kramers-Kronig relation 

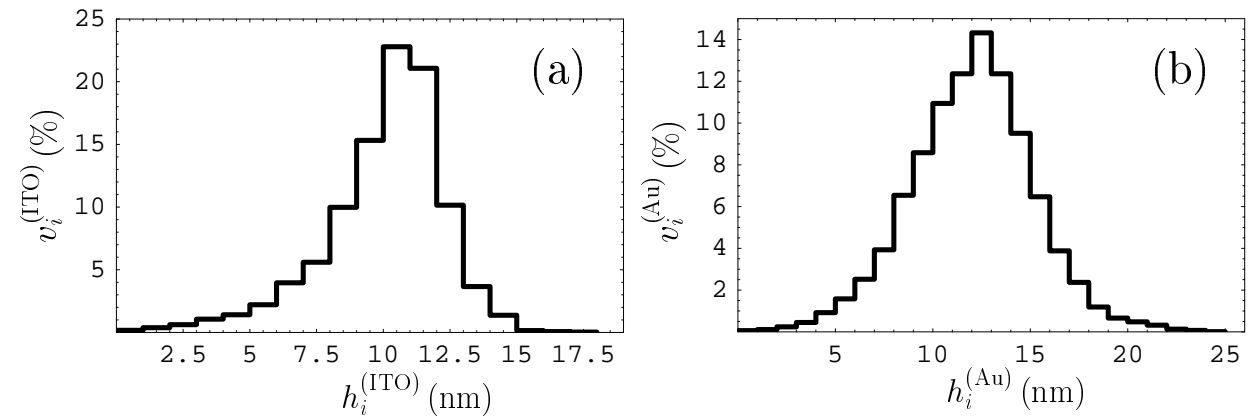

Fig. 6. The fractions of the area $v_{i}$ covered with roughness of heights $h_{i}$ for (a) ITO and (b) Au surfaces.

from the tabulated optical data 34 extrapolated to low frequencies using the imaginary part of the Drude dielectric permittivity with $\omega_{p}=9.0 \mathrm{eV}$ and $\gamma=0.035 \mathrm{eV}$. It was recently shown 35 that such extrapolation nicely fits the optical data measured over a wide region of frequencies.

Using an AFM we have measured the roughness profiles on both the ITO sample and the Au-coated sphere. Specifically, the fractions of area $v_{i}^{(\text {ITO })}$ with heights $h_{i}^{(\mathrm{ITO})}$ (where $i=1,2, \ldots, 18$ ) on an ITO film and $v_{i}^{(\mathrm{Au})}$ with heights $h_{i}^{(\mathrm{Au})}$ on an Au sphere (where $i=1,2, \ldots, 25$ ) were determined [see Figs. 6(a) and 6(b), respectively]. These heights are measured from the absolute minimum level on the respective test body $h_{1}^{(\mathrm{ITO})}=h_{1}^{(\mathrm{Au})}=0$. The zero roughness levels on ITO and Au surfaces, relative to which the mean values of the roughness are equal to zero, 112 take the values $H_{0}^{(\mathrm{ITO})}=9.54 \mathrm{~nm}$ and $H_{0}^{(\mathrm{Au})}=11.51 \mathrm{~nm}$. This information allows computations of the Casimir force with account of surface roughness.

\section{Comparison Between Experiment and Theory}

The Casimir force $F$ acting between a smooth Au-coated sphere and a smooth ITO film deposited on a quartz plate was calculated by using the Lifshitz theory 17 adapted ${ }^{1}$ for a four-layer planar system (Au-vacuum-ITO-quartz) and the proximity force approximation. The latter is valid $\sqrt{36}$ for the experimental parameters and leads to only a very small error of about $a / R \sim 0.1 \%$. The thicknesses of the Au layer and the quartz plate are quite sufficient to model them as semispaces. In this way the force $F$ was found as a function of separation using the dielectric permittivities specified in Sec. 2.2. Computations were performed at the laboratory temperature equal to $2^{\circ} \mathrm{C}$. To obtain the theoretical Casimir forces $F^{\text {theor }}$ acting between rough surfaces we have averaged $F$ over the roughness profiles shown in Fig. 6(a,b) using the method of geometrical averaging! 12

$$
F^{\text {theor }}(a, T)=\sum_{i=1}^{18} \sum_{k=1}^{25} v_{i}^{(\mathrm{ITO})} v_{k}^{(\mathrm{Au})} F\left(a+H_{0}^{(\mathrm{ITO})}+H_{0}^{(\mathrm{Au})}-h_{i}^{(\mathrm{ITO})}-h_{k}^{(\mathrm{Au})}, T\right) .
$$


Although this method is an approximate one, at short separations it leads to the same results for the roughness corrections to the Casimir force arising due to stochastic roughness as the more fundamental scattering approach. ${ }^{37}$ In our experiment, the roughness correction reaches the maximum value of $2.2 \%$ at $a=60 \mathrm{~nm}$ and becomes less than $1 \%$ and $0.5 \%$ at separations $a \geq 90 \mathrm{~nm}$ and $a \geq 116 \mathrm{~nm}$, respectively. When separation increases to about the correlation length of surface roughness (which is equal to a few hundred nanometers), the method of geometrical averaging underestimates the roughness correction. In this separation region the scattering approach is more exact. Note, however, that at such large separations the effect of roughness in negligibly small and can be disregarded. Thus, for practical purposes the method of geometrical averaging is quite sufficient when dealing with stochastic roughness (for periodically corrugated boundary surfaces with sufficiently small period it is necessary to use the scattering approach to obtain good agreement between experiment and theory $38 \mid 39$

The computational results for $F^{\text {theor }}$ as a function of $a$ are shown by the pairs of solid lines in the main fields of Fig. 3(a) for the untreated sample and Fig. 3(b) for the UV-treated sample. Two different lines correspond to different extrapolations of $\operatorname{Im} \varepsilon^{(\mathrm{ITO})}$ to high frequencies indicated in Fig. 4(a,b) by the short-dashed lines. We emphasize that the two solid lines in Fig. 3(a) were computed with the dielectric permittivity shown by the two solid lines in Fig. 5(a), i.e., with included contribution of free charge carriers of the untreated sample. The theoretical lines in Fig. 3(a) form the theoretical band which is in very good agreement with the experimental data indicated as crosses. This result is rather natural and confirms that the optical properties of the ITO film were measured correctly.

A different situation arises with Fig. 3(b) where the theoretical forces were computed with the dielectric permittivity shown by the two solid lines in Fig. 5(b), i.e., with the contribution of free charge carriers of the UV-treated sample disregarded. The theoretical band formed by the two solid lines in Fig. 3(b) is also in very good agreement with the experimental data indicated as crosses, but the reason for this agreement may seem unclear. The point is that the optical and electric properties of the untreated and UV-treated ITO samples at laboratory temperature are shown to be very close. This suggests the use of the two dashed lines in Fig. 5(b) as the true dielectric permittivity of the UV-treated ITO sample. The computational results obtained in this way are presented by the two dashed lines in Fig. 7(a) where the same experimental data as in Fig. 3(b) are indicated as crosses. As is seen in Fig. 7(a), the obvious natural computational approach results in complete contradiction with the data, whereas the seemingly ad hoc omission of the contribution from free charge carriers brings theory into very good agreement with the data. Note that almost the same computational results are obtained when the free charge carriers are described by the plasma model with the plasma frequency $\omega_{p}=9.0 \mathrm{eV}$ for $\mathrm{Au}$ and with the so-called longitudina $\frac{40}{40}$ plasma frequency $\omega_{p}=1.3 \mathrm{eV}$ for ITO (which is smaller than $\omega_{p}$ used for extrapolation of the measured optical data to lower frequencies). 

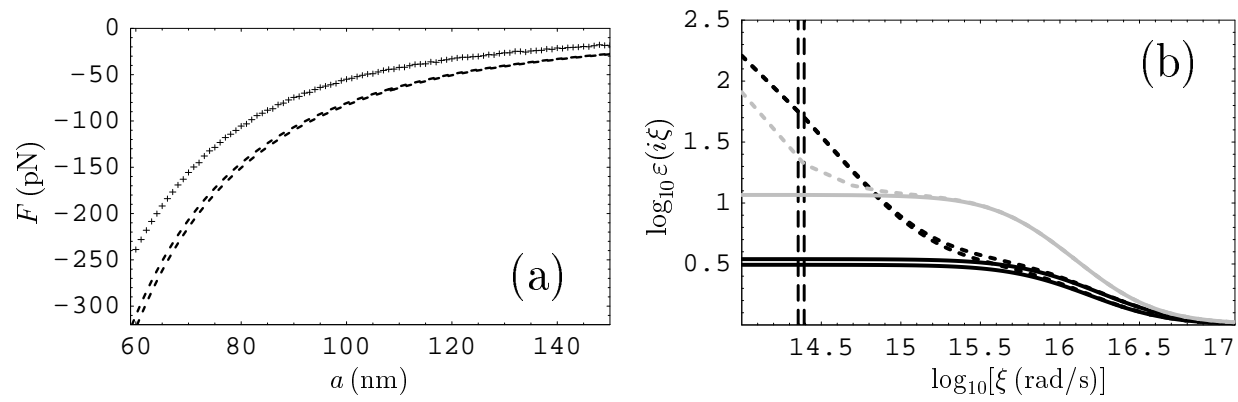

Fig. 7. (a) The mean measured Casimir force as a function of separation (crosses) and the theoretical results computed with included contribution of free charge carriers (two dashed lines) for the UV-treated sample. (b) The pairs of solid black lines and short-dashed black lines show the dielectric permittivity of the UV-treated ITO as a function of imaginary frequency with omitted and included contribution of free charge carriers, respectively. The solid grey and short-dashed grey lines show the dielectric permittivity of $\mathrm{Si}$ in the presence of laser light with omitted and included contribution of free charge carriers, respectively. The vertical long-dashed lines indicate the first Matsubara frequency at $2^{\circ} \mathrm{C}$ (left) and $27^{\circ} \mathrm{C}$ (right).

One way of looking at the decrease in the magnitude of the Casimir force under the UV treatment is in terms of a phase transition. It can be assumed that the UV treatment of the ITO results in the Mott-Anderson transition from a metallic to dielectric state without noticeable change of the optical and electrical properties at room temperature. Specifically, the resistivity of both the untreated and UV-treated samples was measured and shown to be unchanged. The assumption of a phase transition finds support in the observation that the UV treatment of ITO leads to a lower mobility of charge carriers. $\frac{41}{4}$ The dielectric nature of the UV-treated ITO can be verified by the investigation of its electrical properties at low temperature. If the phase transition to dielectric state has occurred, the conductivity of the ITO sample should vanish with temperature. Then the present experiment becomes comparable with other experiments in the Casimir physics which also confirm that the contribution of free charge carriers of dielectric materials should be disregarded. We discuss this point in more detail in the next section.

\section{Phase Transition from Metallic to Dielectric State and Reduction in the Casimir Force}

In the experiment $14 \mid 15$ the difference in the Casimir force between an Au-coated sphere and Si plate in the presence and in the absence of laser pulses on the plate has been measured at room temperature. In the absence of a laser pulse Si was in dielectric state with the density of charge carriers $n \approx 5 \times 10^{14} \mathrm{~cm}^{-3}$. In the presence of a laser pulse $\mathrm{Si}$ was in metallic state because the density of charge carriers was up to 5 orders of magnitude higher. Thus, in the presence of laser pulse charge carrier density was higher than the critical value $n_{c r}$ and in the absence of laser pulse $\mathrm{Si}$ had $n \ll n_{c r}$. The theoretical results for the difference Casimir force computed using the Lifshitz theory were found to be in agreement with experimental data 
only when the free charge carriers in dielectric Si (i.e., in the absence of laser pulse) were disregarded. This is similar to the present experiment. The measured difference in the Casimir forces before and after the phase transition was determined by the actual dielectric permittivity of metallic Si. However, to achieve agreement with the data, the dielectric $\mathrm{Si}$ was replaced with some perfect isolator of zero conductivity, as done with the UV-treated ITO.

In spite of some similarity, there are also differences between the two experiments. In the experiment with ITO the dielectric permittivities of the untreated and UV-treated samples are almost the same. As to the Si plate, the contributions of charge carriers in the absence and in the presence of laser light are quite different because charge carrier densities differ by almost 5 orders of magnitude. One more dissimilarity is that in the experiment with an illuminated Si only a few percent increase in the magnitude of the Casimir force was achieved in the presence of laser pulse (for instance, a $3.8 \%$ and $5.6 \%$ increase at $a=100$ and $150 \mathrm{~nm}$, respectively ${ }^{4}$ ). This is much smaller than the reduction in the force magnitude observed in the ITO sample after the UV treatment.

As to the first difference between the two experiments, our measurement with the UV-treated ITO is really the first demonstration of the significant decrease in the magnitude of the Casimir force with no apparent change of dielectric permittivity. However, if the experiment $14 \mid 15$ with an illuminated Si would be repeated with a sample having the density of charge carriers $n$ in the dark phase only slightly smaller than $n_{c r}$, almost the same measurement results, as in Refs. 14 and 15, are expected. In this case a few percent difference in the Casimir forces would be achieved with almost no change in the dielectric permittivity. A similar effect of the change in Casimir force with almost no change of dielectric permittivity can be also observed in the proposed experiment with a patterned plate $\frac{42}{2}$ consisting of two halves made of semiconductor with $n$ only slightly smaller than $n_{c r}$ and $n$ only slightly larger than $n_{c r}$ (i.e., in the dielectric and metallic states, respectively).

The second difference between the two experiments mentioned above is simply explained by the different materials chosen. In Fig. 7(b) we plot the dielectric permittivity of the UV-treated ITO with omitted (the two solid black lines) and included (the two short-dashed black lines) free charge carriers as a function of imaginary frequency on a double logarithmic scale. In the same figure the solid grey and short-dashed grey lines show the permittivity of dielectric Si with omitted contribution of charge carriers and metallic $\mathrm{Si}$ in the presence of laser light, respectively. As is seen in Fig. 7(b), the inclusion of free charge carriers leads to relative increase of the dielectric permittivity calculated at the respective first Matsubara frequencies by a factor of about 17 for ITO and by a much smaller factor of about 1.8 for $\mathrm{Si}$. This explains why using the UV-treated ITO allows one to obtain so large a reduction in the magnitude of the Casimir force as reported here. 


\section{Conclusions and Discussion}

In this paper we presented additional experimental and theoretical information on the recently observed effect of reduction in the Casimir force without change of dielectric permittivity 18 The reduction occurs after the ITO plate interacting with Au sphere undergoes UV treatment. In so doing the dielectric permittivity of ITO measured by means of ellipsometry both before and after the UV treatment remains almost the same. We have shown that this effect can be described in the framework of the Lifshitz theory if the contribution of free charge carriers in the dielectric permittivity of the UV-treated sample is omitted. Although the presented facts may seem paradoxical, they find a natural explanation under the assumption that the UV treatment caused the transition of an ITO sample from metallic to dielectric phase. Such transition can occur without significant changes of the dielectric permittivity at room temperature (for instance, dielectric to metal transition in doped semiconductors with increase of doping concentration above the critical value). Then neglecting the contribution of free charge carriers in the UV-treated ITO becomes similar to the neglect of dc conductivity in dielectric materials in the Lifshitz theory. As discussed above, the inclusion of dc conductivity of dielectric $\mathrm{Si}$ in the absence of laser pulse was found to be in contradiction with measurements of the Casimir force between an Au sphere and Si plate.14]15 In one more experiment it was demonstrated ${ }^{43}$ that the measured Casimir-Polder force between ${ }^{87} \mathrm{Rb}$ atoms and dielectric $\left(\mathrm{SiO}_{2}\right)$ plate is consistent with theoretical predictions with the dc conductivity of $\mathrm{SiO}_{2}$ omitted, but disagree with the theoretical prediction taking dc conductivity into account 44

Thus, several experiments performed with dielectric test bodies including the one considered in this paper support the phenomenological prescription $1|2| 45$ on how to apply the Lifshitz theory to dielectric materials with no contradictions with thermodynamics and experiment (the inclusion of dc conductivity of dielectrics in the Lifshitz theory was shown $\frac{46}{}$ to violate the Nernst heat theorem). According to this prescription, in calculations of dispersion forces between dielectrics and semiconductors of dielectric type free charge carriers should be disregarded. The possibility to obtain significantly different Casimir forces from the test bodies with nearly equal dielectric permittivities, as observed in the present experiment, is an immediate consequence of this prescription (see the discussion of the proposed experiment 42 in Sec. 4). It is obvious, however, that such phenomenological prescriptions require a more fundamental theoretical justification.

Regardless of whether the above theoretical explanation will be confirmed in future, the observed effect of reduction in the Casimir force without change of dielectric permittivity is prospective for applications in nanotechnology, specifically, in connection with problems of lubrication and stiction. The Casimir and van der Waals forces lead to collapse of closely spaced moving parts of nanoelectromechanical systems to the fixed electrodes leading to loss of functionality of devices. Significant reduction in the magnitude of the Casimir force may help to increase the 
output of efficient nanosystems.

\section{Acknowledgments}

This work was supported by the DARPA Grant under Contract No. S-000354 (equipment, A.B., R.C.-G., U.M.), NSF Grant No. PHY0970161 (C.-C.C., G.L.K., V.M.M., U.M.) and DOE Grant No. DEF010204ER46131 (G.L.K., V.M.M., U.M.). G.L.K. and V.M.M. were also partially supported by the DFG Grant BO 1112/201. U.M., G.L.K. and V.M.M. are grateful to the local Orginizing Committee of QFEXT11 (Benasque, Spain) for their kind hospitality.

\section{References}

1. M. Bordag, G. L. Klimchitskaya, U. Mohideen and V. M. Mostepanenko, Advances in the Casimir Effect (Oxford University Press, Oxford, 2009).

2. G. L. Klimchitskaya, U. Mohideen and V. M. Mostepanenko, Rev. Mod. Phys. 81, 1827 (2009).

3. A. W. Rodriguez, F. Capasso and S. G. Johnson, Nature Photon. 5, 211 (2011).

4. G. L. Klimchitskaya, U. Mohideen and V. M. Mostepanenko, Int. J. Mod. Phys. B 25, 171 (2011).

5. B. W. Harris, F. Chen and U. Mohideen, Phys. Rev. A 62, 052109 (2000).

6. R. S. Decca, D. López, E. Fischbach, G. L. Klimchitskaya, D. E. Krause and V. M. Mostepanenko, Phys. Rev. D 75, 077101 (2007).

7. R. S. Decca, D. López, E. Fischbach, G. L. Klimchitskaya, D. E. Krause and V. M. Mostepanenko, Eur. Phys. J. C 51, 963 (2007).

8. F. Chen, U. Mohideen, G. L. Klimchitskaya and V. M. Mostepanenko, Phys. Rev. A 72, 020101(R) (2005).

9. F. Chen, U. Mohideen, G. L. Klimchitskaya and V. M. Mostepanenko, Phys. Rev. A 74, 022103 (2006).

10. F. Chen, G. L. Klimchitskaya, V. M. Mostepanenko and U. Mohideen, Phys. Rev. Lett. 97, 170402 (2006).

11. S. de Man, K. Heeck, R. J. Wijngaarden and D. Iannuzzi, Phys. Rev. Lett. 103, 040402 (2009).

12. S. de Man, K. Heeck and D. Iannuzzi, Phys. Rev. A 82, 062512 (2010).

13. G. Torricelli, I. Pirozhenko, S. Thornton, A. Lambrecht and C. Binns, Europhys. Lett. 93, 51001 (2011).

14. F. Chen, G. L. Klimchitskaya, V. M. Mostepanenko and U. Mohideen, Optics Express 15, 4823 (2007).

15. F. Chen, G. L. Klimchitskaya, V. M. Mostepanenko and U. Mohideen, Phys. Rev. B 76, 035338 (2007).

16. G. Torricelli, P. J. van Zwol, O. Shpak, C. Binns, G. Palasantzas, B. J. Kooi, V. B. Svetovoy and M. Wuttig, Phys. Rev. A 82, 010101(R) (2010).

17. E. M. Lifshitz and L. P. Pitaevskii, Statistical Physics, Pt. II (Pergamon, Oxford, 1980).

18. C.-C. Chang, A. A. Banishev, G. L. Klimchitskaya, V. M. Mostepanenko and U. Mohideen, Phys. Rev. Lett. 107, 090403 (2011).

19. V. B. Bezerra, G. L. Klimchitskaya and V. M. Mostepanenko, Phys. Rev. A 66, 062112 (2002).

20. F. Chen and U. Mohideen, Rev. Sci. Instrum. 72, 3100 (2001). 
21. H. E. Grecco and O. E. Martinez, Appl. Opt. 41, 6646 (2002).

22. W. R. Smythe, Electrostatics and Electrodynamics (McGraw-Hill, New York, 1950).

23. A. A. Banishev, C.-C. Chang and U. Mohideen, Int. J. Mod. Phys. A 26, 3900 (2011); Int. J. Mod. Phys.: Conf. Ser. 3, 497 (2011).

24. H.-C. Chiu, C.-C. Chang, R. Castillo-Garza, F. Chen and U. Mohideen, J. Phys. A 41, 164022 (2008).

25. W. J. Kim, M. Brown-Hayes, D. A. R. Dalvit, J. H. Brownell and R. Onofrio, Phys. Rev. A 78, 020101(R) (2008).

26. R. S. Decca, E. Fischbach, G. L. Klimchitskaya, D. E. Krause, D. López, U. Mohideen and V. M. Mostepanenko, Phys. Rev. A 79, 026101 (2009).

27. W. J. Kim, M. Brown-Hayes, D. A. R. Dalvit, J. H. Brownell and R. Onofrio, Phys. Rev. A 79, 021102 (2009).

28. R. S. Decca, E. Fischbach, G. L. Klimchitskaya, D. E. Krause, D. López, U. Mohideen and V. M. Mostepanenko, Int. J. Mod. Phys. A 26, 3930 (2011); Int. J. Mod. Phys.: Conf. Ser. 3, 527 (2011).

29. W. J. Kim, A. O. Sushkov, D. A. R. Dalvit and S. K. Lamoreaux, Phys. Rev. A 81, 022505 (2010).

30. H. Fujiwara and M. Kondo, Phys. Rev. B 71, 075109 (2005).

31. G. E. Jellison, Jr. and F. A. Modine, Appl. Phys. Lett. 69, 371 (1996).

32. http://www.jawoollam.com

33. L. Bergström, Adv. Coll. Interface Sci. 70, 125 (1997).

34. Handbook of Optical Constants of Solids, ed. E. D. Palik (Academic, New York, 1985).

35. G. Bimonte, Phys. Rev. A 83, 042109 (2011).

36. B. Geyer, G. L. Klimchitskaya and V. M. Mostepanenko, Phys. Rev. A 82, 032513 (2010).

37. P. A. Maia Neto, A. Lambrecht, and S. Reynaud, Phys. Rev. A 72, 012115 (2005).

38. H.-C. Chiu, G. L. Klimchitskaya, V. N. Marachevsky, V. M. Mostepanenko and U. Mohideen, Phys. Rev. B 80, 121402(R) (2009).

39. H.-C. Chiu, G. L. Klimchitskaya, V. N. Marachevsky, V. M. Mostepanenko and U. Mohideen, Phys. Rev. B 81, 115417 (2010).

40. I. Hamberg and C. G. Granqvist, J. Appl. Phys. 60, R123 (1986).

41. C. N. Li, A. B. Djurišić, C. Y. Kwong, P. T. Lai, W. K. Chan and S. Y. Liu, Appl. Phys. A 80, 301 (2005).

42. R. Castillo-Garza, C.-C. Chang, D. Jimenez, G. L. Klimchitskaya, V. M. Mostepanenko and U. Mohideen, Phys. Rev. A 75, 062114 (2007).

43. J. M. Obrecht, R. J. Wild, M. Antezza, L. P. Pitaevskii, S. Stringari and E. A. Cornell, Phys. Rev. Lett. 98, 063201 (2007).

44. G. L. Klimchitskaya and V. M. Mostepanenko, J. Phys. A: Math. Theor. 41, 312002(F) (2008).

45. G. L. Klimchitskaya, J. Phys.: Conf. Ser. 161, 0112002 (2009).

46. B. Geyer, G. L. Klimchitskaya and V. M. Mostepanenko, Phys. Rev. D 72, 085009 (2005). 